\title{
A general data dependence analysis for parallelizing compilers
}

\author{
Jing Zhou • Guosun Zeng
}

Published online: 19 March 2008

(C) Springer Science+Business Media, LLC 2008

\section{Erratum to: J Supercomput DOI 10.1007/s11227-007-0168-7}

The authors wish to correct their article with the following information:

Affiliations

Jing Zhou ${ }^{1,2}$ and Guosun Zeng ${ }^{1}$

${ }^{1}$ Department of Computer Science and Technology, Tongji University, Shanghai, 201804, China

${ }^{2}$ Department of Statistics, University of California at Berkeley, Berkeley, CA 94720, USA

Acknowledgments

This project was supported by the 863 program under grants 2007AA01Z425 and 973 program under grants 2007CB316502, and the National Natural Science Foundation of China under grants 90718015 and 60673157.

The online version of the original article can be found under doi:10.1007/s11227-007-0168-7.

J. Zhou $(\bowtie) \cdot$ G. Zeng

Department of Computer Science and Technology, Tongji University, Shanghai 201804, China e-mail: daisy883@gmail.com

J. Zhou

Department of Statistics, University of California at Berkeley, Berkeley, CA 94720, USA

e-mail: jingzhou@stat.berkeley.edu 